\title{
Remote Facilitation of Soleus H-reflex Induced by Clenching on Occlusal Stabilization Appliances
}

\author{
Naka Shinji*, DDS, Takahashi Toshiyuki**, DDS, PhD, Sumita Yosuke*, DDS, PhD, \\ Ueno Toshiaki*, DDS, PhD and Ohyama Takashi***, DDS, PhD \\ *Department of Sports Medicine and Dentistry, Graduate School, Tokyo Medical and Dental University \\ **Department of Removable Prosthodontics, Graduate School of Dental Medicine Hokkaido University \\ ***Department of Removable Prosthodontics, Graduate School, Tokyo Medical and Dental University
}

\section{Clinical significance}

Prosthodontists routinely use intraoral splints such as stabilization appliances and repositioning appliances to treat patients with temporomandibular disorders. This study demonstrated that spinal cord excitability modulated during clenching when using stabilization appliances, indicating that these appliances could influence the general motor system in humans.

\section{ABSTRACT}

Purpose: The purpose of this study was to examine the change in spinal cord excitability in a leg muscle during maximal voluntary clenching on a stabilization appliance.

Materials and Methods: The $\mathrm{H}$ reflex of the soleus muscle was elicited in five healthy male volunteers by electrical stimulation of the tibial nerve under the following five dental occlusal conditions: mandibular rest position ( $\mathrm{RP}$ : control), maximum voluntary clenching in the intercuspal position (IP), maximum voluntary clenching on a hard acrylic stabilization appliance ( $\mathrm{H}-\mathrm{SA})$, maximum voluntary clenching on a soft stabilization appliance (S-SA) and maximum voluntary clenching on a very soft stabilization appliance (VS-SA).

Results : The soleus H reflex modulations in the IP, H-SA, S-SA and VS-SA conditions were 117.6\%, $119.5 \%, 126.2 \%$ and $133.6 \%$, respectively. There was a significant difference between IP and S-SA, and IP and VS-SA $(p=0.0001-0.0226)$; however, there was no significant difference between IP and $\mathrm{H}-\mathrm{SA}(\mathrm{p}=0.3412-0.9940)$. Significant positive correlations between the magnitude of the soleus $\mathrm{H}$ reflex and the masseter electromyogram activity were found in each subject $(r=0.670-0.862, p<0.05)$. Conclusion: This study demonstrated that the spinal cord excitability in the motor pathway of a leg muscle was considerably raised during clenching on a soft stabilization appliance compared to a hard stabilization appliance, suggesting that a powerful biting action on a resilient intraoral appliance strongly influences the motor system in humans.

\section{Key words}

$\mathrm{H}$ reflex, soleus muscle, remote facilitation, teeth clenching, stabilization appliance

Corresponding author : Naka Shinji

1-5-45 Yushima, Bunkyo-ku, Tokyo 113-8549, Japan

Tel and Fax : +81-3-5803-5867

E-mail : naka.spmd@tmd.ac.jp

Received on January 22, 2004/Accepted on April 7, 2004 


\section{INTRODUCTION}

Since the field of sports dentistry began in the middle of the 20 th century, many researchers $^{1-9)}$ have become interested in improving muscle strength and movements, such as longdistance running ${ }^{10)}$ and golf swings ${ }^{11)}$, through the use of special intraoral appliances such as occlusal splints, mandibular orthopedic repositioning appliances, and mouthguards. However, this issue still appears to be controversial ${ }^{10,12-20)}$.

As sports medicine and dentistry developed, there has been renewed interest in the possible relation of oral motor function itself on physical motor performance. In our previous neurophysiological studies ${ }^{21-23)}$ using the Hoffman reflex, which can be used to assess the excitability of spinal alfa motoneurons while also reflecting transmission efficiency (i.e., presynaptic inhibition) in Ia afferent synapses ${ }^{24)}$, the $\mathrm{H}$ reflex of the soleus, the pretibial muscles, the flexor carpi radialis and the extensor carpi radialis were facilitated in association with voluntary teeth clenching in humans. It was also demonstrated that there was a positive correlation between the extent of facilitation of the reflex and the magnitude of the electromyographic activity of the masseter muscle. Additionally, reciprocal Ia inhibition from pretibial muscles to the soleus muscle was depressed with voluntary teeth clenching, indicating that teeth clenching exerts nonreciprocal tonic facilitation of the $\mathrm{H}$ reflex of the ankle extensors and flexors. It is accordingly suggested that teeth clenching contributes to the improved stability of posture and stance rather than smoothness of movements. These suggestions were fully supported by biomechanical research ${ }^{7,25-28)}$ using isometric and/or isokinetic dynamometry, which has shown that teeth clenching could lead to an increase in the static maximal strength of appendage muscles but no increase in dynamic output.

Muscles produce force and movements that vary in magnitude, speed and precision ${ }^{29)}$. The variety of force profiles and movements depends on both peripheral factors, such as the anatomy and fiber composition of the muscles, and central factors, such as motor commands. Regardless of the sophistication of muscles in their design and composition, they act in response to neural commands to produce the required range of motor output. The motor system in humans is comprised of two neural centers that generate and regulate the motor commands; one is the motor cortex, and the other is the spinal cord. If the ameliorative effect on motor ability of wearing intraoral appliances is a real phenomenon, the spinal motoneuron excitability at least should be raised, because the spinal cord is a lower motor center that communicates with the muscle directly in the neuromuscular circuit. However, it is uncertain whether motor neuron excitability at the spinal cord level modulates during voluntary clenching on oral appliances or not.

Therefore, we studied the change of the $\mathrm{H}$ reflex in a leg muscle during maximal voluntary clenching on stabilization appliances in an effort to resolve the controversy mentioned above. Three different degrees of hardness of stabilization appliances were tested in the present study: one was made with hard acrylic resin material, and the others were made of the soft elastic material used in sports mouthguards.

\section{MATERIALS AND METHODS}

\section{Subjects}

The experiments were performed on five healthy male volunteers, aged 27-32 years, all of whom had given their informed consent to this study, which was approved by the intramural committee for experiments on human subjects. All of them had a full complement of natural 
teeth, at least up to the second molars, with a healthy periodontium; there were no signs and symptoms of craniomandibular disorders and no functional disorders of the body.

\section{Stabilization Appliances}

Each subject received three stabilization appliances custom made from different materials: (1) a standard hard stabilization appliance with dental acrylic resin (Acron ${ }^{\circledR}$, GC, Japan); (2) a soft stabilization appliance with resilient polyolefin material for sports mouthguards (MG 21 ${ }^{\circledR}$, Molten Medical, Japan; JIS-A hardness 82 ) ; and (3) a very soft stabilization appliance with resilient polyolefin material for sports mouthguards $\left(\mathrm{MG} 21^{\circledR}\right.$, Molten Medical, Japan ; JIS-A hardness 72). Alginate impressions of the dental arches, and interocclusal recording with addition reaction polyvinylsiloxane material (Exabite $\mathrm{II}^{\circledR}, \mathrm{GC}$, Japan) and a face-bow were obtained. The dental stone casts were mounted onto a semi-adjustable articulator (Denar Mark II Articulator ${ }^{\circledR}$, Fort Collins, CO, USA) using the face-bow transfer and split cast method. All of the stabilization appliances were fabricated on a maxillary stone cast model using the lost wax technique and finished so that they were identical in appearance. The appliances covered all of the maxillary teeth, except for the third molars, and had the same occlusal thickness in accordance with the mandibular rest position of each subject and even occlusal contacts during light clenching in the centric position for all opposing teeth.

\section{Electromyography}

The electromyographic (EMG) activities of the right soleus muscle and the right and left masseter muscles were recorded with bipolar surface $\mathrm{Ag}-\mathrm{AgCl}$ electrodes (diameter $8.0 \mathrm{~mm}$ ). After careful preparation of the skin (shaving, abrasion and cleaning with alcohol), the elec- trodes were placed $2 \mathrm{~cm}$ apart longitudinally in the middle of the masseter muscles and $3 \mathrm{~cm}$ apart longitudinally in the middle of the soleus muscle. The EMG activities were amplified with conventional differential amplifiers (time constant $0.03 \mathrm{~s}$, high-cut frequency $10 \mathrm{kHz}$ ), and stored on a digital audio data recorder (Sony PC 216 A, Japan ; flat frequency response DC-5.0 $\mathrm{kHz}$ ) along with the stimulus pulses. Hard copies from the data recorder were obtained using a thermal array recorder (RJA-1300, Nihon Kohden, Japan ; flat frequency response $\mathrm{DC}-10 \mathrm{kHz})$.

\section{H reflex Recordings}

Each subject was comfortably seated in a reclining chair, constantly keeping his foot on an immobile footplate with the angle of the knee and foot joints on both sides at approximately $120^{\circ}$ and $100^{\circ}$, respectively. The subject's head reclined on a headrest, and his forearm and hand on an armrest, because the soleus H reflex is influenced by the posture of the head and upper extremities $^{30)}$. The armrest and the footplate also served to keep the muscles of the arm and leg relaxed during the resting period of the experiment to prevent the subject from becoming fatigued.

The $\mathrm{H}$ reflex and direct muscle responses (M wave) of the soleus were evoked with a surface electrode (diameter $8.0 \mathrm{~mm}$ ) positioned in the right popliteal fossa over the tibial nerve as a cathode and a silver plate $(35.0 \times 45.0 \mathrm{~mm})$ placed on the patellar region as the anode. A ring silver plate was placed on the skin between the stimulating and recording electrodes as the ground electrode to reduce artifacts due to the spreading of the stimulating current along the surface of the skin. A 1-ms stimulating pulse was used, the intensity of which was adjusted to about $30 \%$ of the maximum $\mathrm{M}$ wave, which was similar to those in our previous reports ${ }^{21,22)}$. At this stimulus intensity, a small $\mathrm{M}$ wave was 
obtained together with the $\mathrm{H}$ reflex and was used to monitor the stability of the stimulating condition. It had already been shown that repeated stimulation of the tibial nerve induced a depression of the soleus $\mathrm{H}$ reflex for as long as $10 \mathrm{~s}^{31-33)}$. However, it was reported that virtually no inhibition was detected at an inter-stimulus interval of $>8 \mathrm{~s}^{31)}$. As the $\mathrm{H}$ reflex is also known to be strongly affected by psychic condition ("motor set") ${ }^{34)}$, the inter-stimulus interval was set from 8.0 to $12.0 \mathrm{~s}$ in $0.5^{-\mathrm{S}}$ steps in a random sequence with a random pulse generator.

\section{Experimental Procedures}

The experiment was carried out under the following five conditions: 1) no clenching, with the jaw relaxed in the rest position ( $\mathrm{RP}:$ control) ;2) maximal voluntary clenching of the teeth in the intercuspal position (IP) ; 3) maximal voluntary clenching on the standard hard stabilization appliance (H-SA) ; 4) maximal voluntary clenching on the soft stabilization appliance (S-SA); and 5) maximal voluntary clenching on the very soft type stabilization appliance (VS-SA).

The full-wave rectified, integrated EMG in the right masseter muscle was monitored as an indicator of the strength of clenching. Two symbols were shown on the computer display about $1 \mathrm{~m}$ in front of the subject, as shown in Fig. 1. Two parallel vertical straight lines in the top half of the display (serving as the "target") indicated the maximum contraction of the masseter muscle. The space between the two lines indicated the strength of the muscle contraction as the mean amplitude of the integrated EMG calculated from a 3 -s period during the maximum voluntary contraction of the masseter muscle measured prior to test trials; the distance of the two lines from the center of the space was $\pm 5 \%$. The arrow in the bottom half of the display (indicating the force) continuously showed the

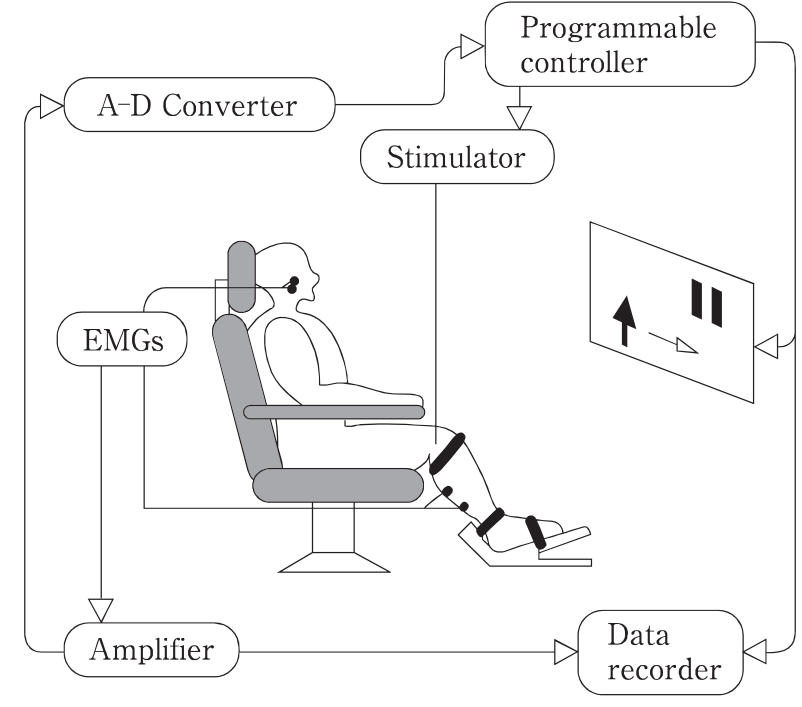

Fig. 1 Diagram of experimental setup The pair of vertical lines on the screen in front of the subject was the target cursor. The arrow moved from left to right accord ing to the degree of the masseter muscle contraction

instantaneous amplitude of a full-wave rectified, integrated EMG during the test trial. The left and right ends of the display corresponded to the 0 and $100 \%$ levels of the voluntary muscle contraction, respectively. The force arrow moved to the right as the masseter muscle contracted.

The subjects were instructed to relax the muscles of their extremities during the test trials. They were initially instructed to maintain their jaws in the rest position when neither symbol was shown in the display. In the control trials, neither symbol was displayed on the screen. Both the "target" positioned at the right side in the screen and the force arrow at the left end showing a 0 level of contraction of the masseter muscle were displayed simultaneously in the test trials (IP, H-SA, S-SA and VS-SA). When these cursors appeared after the sound of a beep signaling "go", the subject was instructed to clench his teeth as much as possible and move the force arrow to the target as quickly as possible and to maintain this clenching force for about $3 \mathrm{~s}$. Tibial nerve stimulation to evoke the soleus $\mathrm{H}$ 
reflex was randomly given at different intervals after the masseter EMG onset.

One session consisted of 10 trials (five control trials and five trials for each condition), and a series of 8 sessions ( 2 sessions per condition) were performed in one day with a 5 -min rest interval between the sessions.

\section{Data Processing and Analysis}

The magnitude of the $\mathrm{H}$ reflex was defined as the peak-to-peak amplitude, that is, the difference between the maximum and minimum values within a time window from about 20-50 ms after the electrical stimulation of the tibial nerve. The amplitudes of the $\mathrm{H}$ reflex were further normalized in relation to the control condition in the same session to quantify the modulations of the $\mathrm{H}$ reflex ; the magnitude of the $\mathrm{H}$ reflex was expressed as a percentage of the control. The magnitude of the jaw muscle activity was defined as the integrated EMGs of the left and right masseter muscles during $500 \mathrm{~ms}$ before and after electrical stimulation of the tibial nerve. The mean integrated EMG activities in conditions RP, H-SA, S-SA and VS-SA were normalized in relation to the IP condition and were expressed as a percentage of the IP condition.

The results were statistically analyzed using one-way analysis of variance (one-way ANOVA) followed by the Fisher PLSD post-hoc test $(\mathrm{p}<0.05)$. The possible relationship between the $\mathrm{H}$ reflex modulation and the masseter EMG activities was assessed using the Pearson's correlation coefficient.

\section{RESULTS}

Table 1 shows the integrated EMG of the masseter muscles for each test condition of all five subjects tested. The grand mean masseter EMG activities in the H-SA, S-SA and VS-SA conditions were $109.7 \%, 120.0 \%$ and $127.5 \%$, respectively. There were statistically significant increases in the EMG amplitude of the masseter muscles for the S-SA and VS-SA conditions compared with the IP condition $(p=0.008$ and 0.001 , respectively) while the $\mathrm{H}-\mathrm{SA}$ condition did not significantly increase $(p=0.0656)$.

Figure 2 illustrates the soleus EMG evoked successively by electrical stimulation of the tibial nerve in the same subject. As seen in the superimposed records in Fig. $2 \mathrm{~F}$ (superimposed EMGs of RP, IP, H-SA, S-SA and VS-SA), every component wave composing the soleus $\mathrm{H}$ reflex increased in amplitude in the IP, H-SA, S-SA and VS-SA conditions. There was no difference in either the waveform or amplitude of the M wave in Fig. $2 \mathrm{~F}$, indicating that there was no change in intensity of the stimulus applied to the tibial nerve during maximal voluntary clenching under each condition compared with the control condition.

Table 2 shows the soleus $\mathrm{H}$ reflex modulation in each of the five subjects, and Fig. 3 illustrates the grand mean amplitudes of the soleus $\mathrm{H}$ reflex of all subjects. The grand means of the soleus $\mathrm{H}$ reflex modulation in the IP, $\mathrm{H}-\mathrm{SA}, \mathrm{S}-$ SA and VS-SA conditions were 117.6\%, 119.5\%,

Table 1 Means and standard deviations of masseter EMG (\% of IP)

\begin{tabular}{cccc}
\hline \hline & H-SA & S-SA & VS-SA \\
\hline Subject 1 & $107.61 \pm 10.99$ & $112.91 \pm 9.01$ & $118.22 \pm 12.79$ \\
Subject 2 & $116.33 \pm 12.43$ & $129.49 \pm 9.01$ & $134.37 \pm 17.04$ \\
Subject 3 & $100.26 \pm 5.88$ & $111.92 \pm 8.91$ & $118.84 \pm 7.21$ \\
Subject 4 & $122.11 \pm 12.22$ & $127.51 \pm 7.44$ & $138.40 \pm 7.73$ \\
Subject 5 & $101.99 \pm 10.36$ & $118.29 \pm 14.88$ & $131.46 \pm 11.71$ \\
\hline Total subjects & $109.66 \pm 2.65$ & $120.02 \pm 9.85$ & $127.46 \pm 4.03$ \\
\hline
\end{tabular}


$126.2 \%$ and $133.6 \%$, respectively. There was a significant difference between IP and S-SA, and IP and VS-SA in each subject $(\mathrm{p}=$ 0.0001-0.0226) ; however, there was no significant difference between IP and $\mathrm{H}-\mathrm{SA}$ ( $\mathrm{p}=$
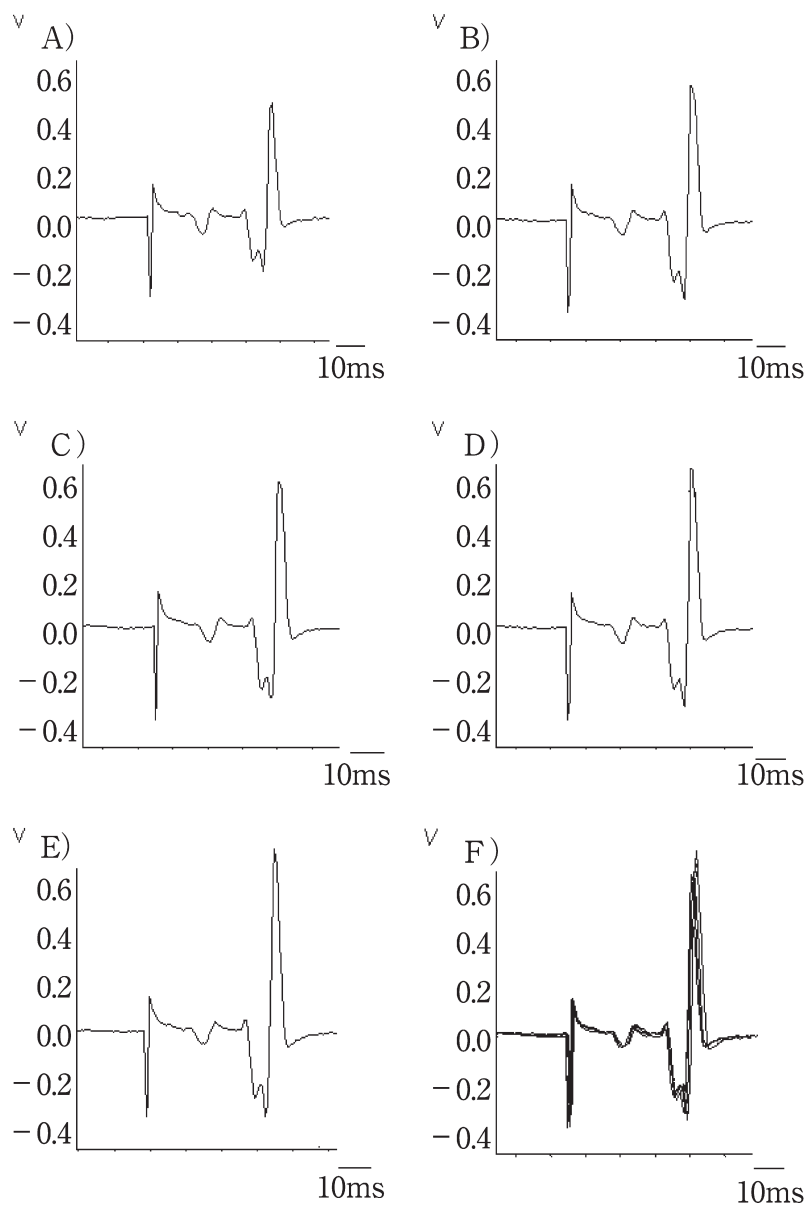

Fig. 2 Modulation of the $\mathrm{H}$ reflex in the soleus muscles during the five conditions in the same subject

A : EMG for RP, B : EMG for IP, C : EMG for H-SA, D : EMG for S-SA, E : EMG for VS-SA, F : Superimposed records of $\mathrm{A}, \mathrm{B}, \mathrm{C}, \mathrm{D}$ and $\mathrm{E}$
$0.3412-0.9940)$.

Table 3 shows the latencies of the soleus $H$ reflex in each subject tested $(n=10$ in each condition). The soleus $\mathrm{H}$ reflex latencies in all the clenching conditions (IP, H-SA, S-SA and VS$\mathrm{SA}$ ) did not change compared with the control trial $(\mathrm{p}=0.0573-0.9996)$.

The time patterns of the soleus $\mathrm{H}$ reflex facilitation of all five subjects tested is shown in Fig. 4. The interval between the test stimulation and the onset of the masseter EMG activity was considered to be time 0 on the abscissa. The $\mathrm{H}$ reflex magnitudes at every $200 \mathrm{~ms}$ interval after the onset of masseter EMG were analyzed in this study. The first interval (0 to $200 \mathrm{ms)}$ was excluded from the analysis because of the lack of available data. The diamond, square, triangle, open circle and filled circle indicate the amplitude of the $\mathrm{H}$ reflex in the IP, $\mathrm{H}_{-}^{-\mathrm{SA}}, \mathrm{S}-\mathrm{SA}, \mathrm{VS}-$ $\mathrm{SA}$ and RP conditions, respectively, as a percent-

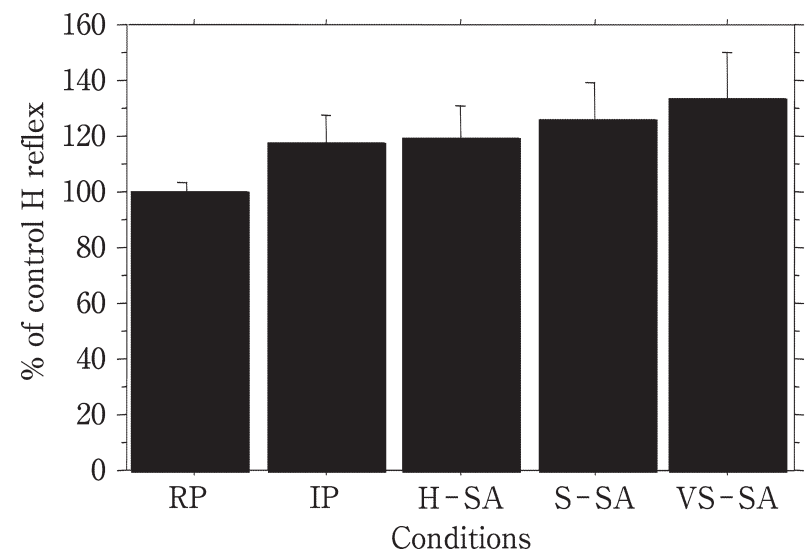

Fig. 3 Histograms showing modulation of soleus $\mathrm{H}$ reflex in all subjects

Bars represent standard deviation

Table 2 Means and standard deviations of facilitation of the soleus H reflex (\% of RP)

\begin{tabular}{ccccc}
\hline \hline & IP & H-SA & S-SA & VS-SA \\
\hline Subject 1 & $119.43 \pm 15.82$ & $119.37 \pm 12.77$ & $129.28 \pm 20.98$ & $139.40 \pm 22.60$ \\
Subject 2 & $119.60 \pm 7.41$ & $124.77 \pm 15.39$ & $129.94 \pm 13.50$ & $139.18 \pm 22.20$ \\
Subject 3 & $114.50 \pm 4.84$ & $114.77 \pm 4.45$ & $119.88 \pm 9.04$ & $122.75 \pm 6.70$ \\
Subject 4 & $121.71 \pm 9.66$ & $125.01 \pm 9.90$ & $129.81 \pm 6.47$ & $137.21 \pm 7.01$ \\
Subject 5 & $112.74 \pm 9.55$ & $113.55 \pm 4.01$ & $122.16 \pm 7.98$ & $129.21 \pm 10.88$ \\
\hline Total subjects & $117.59 \pm 4.06$ & $119.49 \pm 5.03$ & $126.21 \pm 11.59$ & $133.55 \pm 7.95$ \\
\hline
\end{tabular}


Table 3 Means and standard deviations of $\mathrm{H}$ reflex latencies of the soleus $\mathrm{H}$ reflex (ms)

\begin{tabular}{cccccc}
\hline \hline & RP & IP & H-SA & S-SA & VS-SA \\
\hline Subject 1 & $26.50 \pm 0.47$ & $26.85 \pm 0.34$ & $26.90 \pm 0.61$ & $26.40 \pm 0.42$ & $26.40 \pm 0.32$ \\
Subject 2 & $30.60 \pm 0.61$ & $31.70 \pm 0.42$ & $31.70 \pm 0.42$ & $30.65 \pm 0.47$ & $30.45 \pm 0.55$ \\
Subject 3 & $29.20 \pm 0.67$ & $29.40 \pm 0.32$ & $29.20 \pm 0.42$ & $29.40 \pm 0.52$ & $28.75 \pm 0.35$ \\
Subject 4 & $28.20 \pm 0.26$ & $27.90 \pm 0.32$ & $27.75 \pm 0.42$ & $28.05 \pm 0.28$ & $27.55 \pm 0.28$ \\
Subject 5 & $27.05 \pm 0.44$ & $27.00 \pm 0.47$ & $26.85 \pm 0.24$ & $27.25 \pm 0.35$ & $26.85 \pm 0.24$ \\
\hline
\end{tabular}

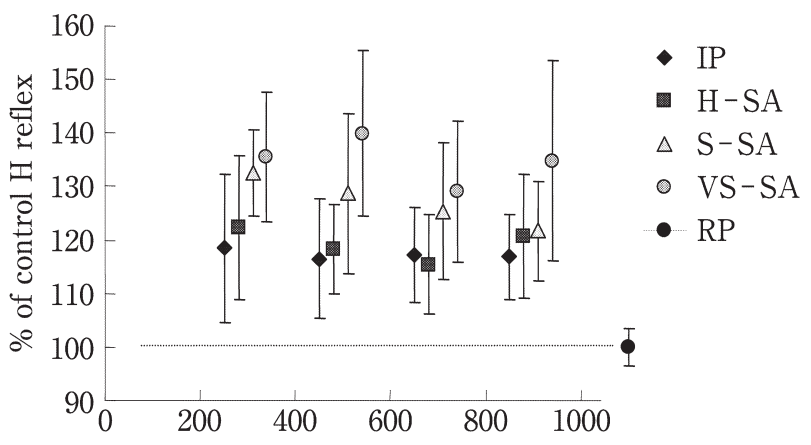

Interval between EMG onset and test stimulus (ms)

Fig. 4 Length of time of modulation of the $\mathrm{H}$ reflex soleus muscles with respect to the onset of masseter muscle EMG

age of the control amplitude at the time when the test stimulus was applied. The vertical bars above and below each symbol represent $+/-1$ SD. The significant and tonic facilitation of the soleus $\mathrm{H}$ reflex in all the IP, H-SA, S-SA and VS-SA conditions was found in all of the intervals compared with the control condition $(p=$ 0.0018-0.0001).

The correlation between the magnitude of the jaw muscle activities and the mean magnitude of the soleus $\mathrm{H}$ reflex in each of the five subjects tested (open circle connected by a straight line) is found in Fig. 5. Significant positive correlations between the magnitude of the soleus $\mathrm{H}$ reflex and the masseter EMG activity were found in each subject $(\mathrm{r}=0.670-0.862, \mathrm{p}<$ 0.05). The filled circles connected with a straight line represent the grand mean amplitudes of all subjects against the amplitudes of the integrated masseter EMG. There was a significant positive correlation between the amplitude of the $\mathrm{H}$ reflex and the jaw muscle EMG activity $(\mathrm{r}=0.719, \mathrm{p}<0.05)$

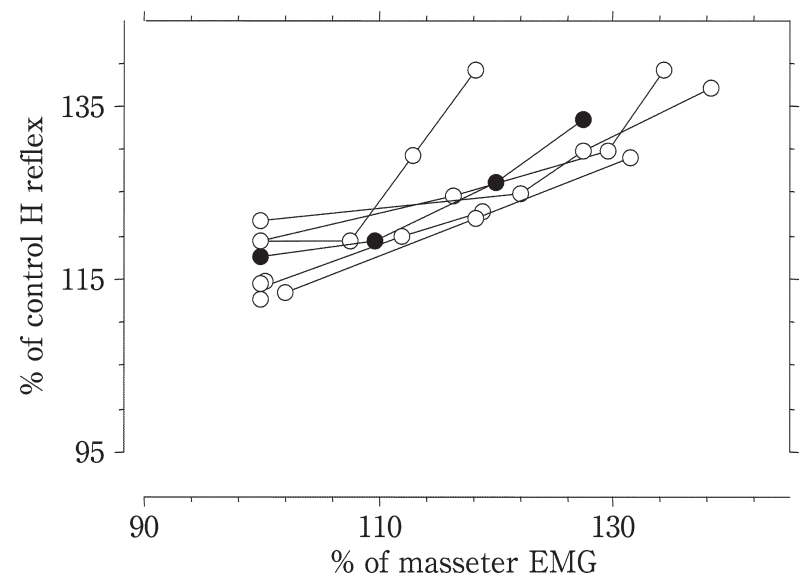

Fig. 5 Correlation between the facilitation of $\mathrm{H}$ reflex and the masseter EMG activities. Data were obtained from all subjects. Each number of the data in IP, H-SA, S-SA and VS-SA was 10

\section{DISCUSSION}

The present study demonstrated that the soleus $\mathrm{H}$ reflex was significantly facilitated during all the clenching conditions (IP, H-SA, S-SA and VS-SA), and the gain of the $\mathrm{H}$ reflex modulated tonically during biting. The $\mathrm{H}$ reflex of the soleus muscle was facilitated during $\mathrm{H}-\mathrm{SA}$ condition at almost the same level as the IP condition; however, the soleus $\mathrm{H}$ reflex was more facilitated during the S-SA and VS-SA conditions than during the IP and $\mathrm{H}-\mathrm{SA}$ conditions. In addition, there was a positive correlation between the amplitude of the soleus $\mathrm{H}$ reflex and the magnitude of the EMG activities of the masseter muscles of the four clenching conditions. The $\mathrm{H}$ reflex latencies of the soleus muscle were not changed among the testing conditions. 


\section{Masseter EMG Activities}

It is well known that the vertical dimension ${ }^{35)}$ and horizontal position ${ }^{36)}$ of the mandible in regard to the maxilla affect masseter EMG activities. Bakke et al. ${ }^{37)}$ also reported that the activity of the masseter muscle during maximal clenching positively correlated to the number of occlusal contacts. Therefore, all three stabilization appliances used in this study were carefully fabricated with the same vertical dimensions, equal horizontal position and even occlusal contacts on the semi-adjustable articulator using the face-bow transfer and check bite techniques; that is, the only difference among these stabilization appliances was the hardness.

There was no significant difference of the masseter EMG activity between the IP and $\mathrm{H}-\mathrm{SA}$ conditions in the normal volunteer subjects of this study. This finding is well supported by previous research ${ }^{38,39)}$. In addition, the masseter muscle activities during maximal biting on soft elastic stabilization appliances (S-SA and VSSA) were significantly greater than during the IP and $\mathrm{H}-\mathrm{SA}$ conditions. This finding is also supported by previous studies. Al Quran and Lyons $^{40)}$ reported that the insertion of a soft splint produced a significant increase in the EMG amplitude of the masseter muscle compared to the insertion of a hard splint. They mentioned that the increase could simply have been because the soft material was more comfortable to bite on. Paphangkorakit and Osborn ${ }^{41}$ have showed that maximum bite force was larger when incising on a soft object (rubber) than on a hard object (acrylic resin) because when the teeth sank into the rubber, the bite force was spread over a larger area, thereby reducing the local stress to the tooth. They suggested that pulpal mechanoreceptors may be used subconsciously to limit the maximum biting force and consciously to detect differences in hardness, although it is well known that periodontal mechanoreceptors play an important role in controlling occlusal biting force.

Concerning the increasing rates of masseter EMG activities during maximal biting of soft appliances, our data on S-SA and VS-SA showed $20 \%$ and $27.5 \%$ increases, respectively. Previous reports have stated that the increasing rates of jaw muscle EMG activities during maximal biting of a soft mouthguard were $32.8-36.3 \%$ compared to the rate that occurred while clenching in the intercuspal position. Collectively, it suggests that maximum voluntary clenching on a soft elastic intraoral appliance may produce an approximately $20-30 \%$ increase in magnitudes of masseter muscle activity.

\section{Possible Neural Basis}

Regarding the neural mechanism of the $\mathrm{H}$ reflex potentiation with the teeth clenching maneuver, several studies ${ }^{21,22)}$ have indicated the involvement of both the supraspinal descending impulses and the peripheral afferent impulses, such as the periodontal mechanoreceptors and muscle spindle.

Recent studies ${ }^{42-44)}$ of the motor-evoked potential (MEP) using the transcranial magnetic stimulation (TMS) technique have revealed that the remote facilitation effect with teeth clenching occurs in the supraspinal area. Sugawara and Kasai ${ }^{42)}$ reported remote facilitation on the cortical site by teeth clenching. Boroojerdi et al. ${ }^{43)}$ found that cortical and subcortical sites contributed to the $\mathrm{H}$ reflex enhancement of hand muscles from teeth clenching, while only subcortical sites were identified in this facilitatory effect on the lower extremity. According to a study by Furubayashi et al. ${ }^{44)}$, the hand motor area was enhanced without spinal cord facilitation at the early phase $(<50 \mathrm{~ms}$ after the onset masseter EMG) of teeth clenching, and spinal facilitation dominated at the late phase $(>50 \mathrm{~ms})$. 
Larson et al. ${ }^{45}$ reported that some muscle spindle afferents (assumed spindle primary afferents) underwent a large, sustained increase in the firing rate during controlled isometric bites in monkeys, but the firing rate was very weakly related to the magnitude of bite forces. Another group of the muscle spindle afferents (assumed spindle secondary afferents) did not increase their firing rate during bite responses. In contrast, the firing frequency of slowly adapting periodontal mechanoreceptive afferents in rabbits increased smoothly with increasing biting force and decreased as the biting force was reduced $^{46)}$. In humans, it was reported that the firing frequency of most periodontal mechanoreceptive afferents increased with the level of force applied to the tooth, resulting in a hyperbolic (i.e., negatively accelerating) relationship between the amplitude of the applied force and the steady-state response ${ }^{47}$. Therefore, the finding that the soleus $\mathrm{H}$ reflex reinforced during clenching in proportion to the strength of the biting force may suggest that periodontal mechanoreceptors play a major role in this facilitation rather than muscle spindle afferents. However, further detailed research will be required to examine the possible involvement of other mechanoreceptors, such as temporomandibular mechanoreceptors, in the interaction between dental occlusion and the general motor system.

\section{Functional Implications}

The results of the present study have clearly demonstrated that the $\mathrm{H}$ reflex of the soleus muscle undergoes a tonic facilitation during voluntary clenching on a stabilization appliance. Additionally, the soleus $\mathrm{H}$ reflex exhibited a marked facilitation while the subject was biting the softer stabilization appliance, which implies that clenching on resilient intraoral appliances, including sports mouthguards, strongly influences the motor system in humans.
Acknowledgements : A part of this study was presented at the 2003 Annual Scientific Meeting of the Korean Academy of Prosthodontics and the 1 st Joint Academic Meeting with the Japan Prosthodontic Society in April 2003, Seoul, Korea.

\section{REFERENCES}

1) Stenger JM. Physiologic dentistry with Notre Dame athletes. Basal Facts 2: 8-18, 1977.

2) Smith SD. Muscular strength correlated to jaw posture and the temporomandibular joint. NY State Dent J 44:278-285, 1978 .

3) Garabee WF Jr. Craniomandibular orthopedics and athletic performance in the long distance runner : A three year study. Basal Facts 4:77-81, 1981.

4) Williams MO, Chaconas SJ, Bader P. The effect of mandibular position on appendage muscle strength. J Prosthet Dent 49:506-507, 1983 .

5) Bates RE Jr, Atkinson WB. The effects on maxillary MORA's on strength and muscle efficiency tests. J Craniomandib Pract 1:37-42, 1983.

6) Verban EM Jr, Groppel JL, Pfautsch EW et al. The effects of a mandibular orthopedic repositioning appliance on shoulder strength. J Craniomandib Pract 2:233-236, 1984

7) Sasaki $Y$, Ueno $T$, Taniguchi $H$ et al. Effect of teeth clenching on isometric and isokinetic strength. J Med Dent Sci 45:23-37, 1998.

8) Wang $\mathrm{K}$, Ueno $\mathrm{T}$, Taniguchi $\mathrm{H}$ et al. Influence on isometric muscle contraction during shoulder adduction by changing occlusal situation. Bull Tokyo Med Dent Univ $43: 1-12,1996$.

9) Taniguchi H, Ueno T, Sasaki $Y$ et al. Influence of MORA on isometric muscle strength of upper appendage during shoulder adduction. J Sports Dent $1: 31-39,1998$

10) Burkett LN, Berstein AK. The effect of mandibular position on strength, reaction time and movement time on a randomly selected population. NY State Dent J 49 : 281-285, 1983.

11) Erget C, Leroy D, Loret A et al. Effect of mandibular orthopedic repositioning appliance on kinematic pattern in golf swing. Int J Sports Med $23: 148-152$, 2002.

12) Greenberg MS, Cohen SG, Springer P et al. Man dibular position and upper body strength : A controlled clinical trial. J Am Dent Assoc 103: 576-579, 1981.

13) Hart DL, Lundquist DO, Davis HC. The effect of vertical dimension on muscular strength. J Othop Sports Phys Ther $3: 57-61,1981$.

14) Burkett LN, Berstein AK. Strength testing after jaw repositioning with a mandibular orthopedic appliance. Phys Sports Med 10:101-107, 1982.

15) Yates JW, Koen TJ, Semenick DM et al. Effect of a mandibular orthopedic repositioning appliance on muscular strength. J Am Dent Assoc 108:331-333, 1984.

16) Shubert MM, Hall R, Guttu RL et al. Changes in shoulder and legs strength in athletes wearing man- 
dibular orthopedic repositioning appliances. J Am Dent Assoc 108:334-337, 1984.

17) Allen ME, Walter $P, M c K a y ~ C$ et al. Occlusal splints (MORA) vs. placebos show no difference in strength in symptomatic subjects: Double blind/ cross-over study. Can J Appl Sport Sci 9 : 148-152, 1984.

18) Parker MW, Pelleu GB Jr, Blank LW et al. Muscle strength related to use of interocclusal splints. Gen Dent 32 : 105-109, 1984.

19) Welch MJ, MacEdington CD, Ritter MR. Muscular strength and temporomandibular joint repositioning. J Orthop Sports Phy Ther 7 :236-239, 1986.

20) Forgione AG, Mehta NR, Westcott WL. Strength and bite, part I : An analytical review. J Craniomandib Pract $9:$ 305-315, 1991.

21) Miyahara $T$, Hagiya $T$, Ohyama $T$ et al. Modulation of human soleus $\mathrm{H}$ reflex in association with voluntary clenching of the teeth. J Neurophysiol 76 : 2033-2040, 1996.

22) Takada Y, Miyahara T, Tanaka T et al. Modulation of $\mathrm{H}$ reflex of pretibial muscles and reciprocal Ia inhibition of soleus muscle during voluntary teeth clenching in humans. J Neurophysiol $83: 2063-2070$, 2000.

23) Takahashi $T$, Ueno $T$, Ohyama $T$ et al. Modulation of $\mathrm{H}$ reflex in the forearm during voluntary teeth clenching in humans. Euro J Appl Physiol 90: 651-653, 2003.

24) Aagaard P, Simonsen EB, Andersen JL. Neural adaptation to resistance training : Change in evoked V-wave and H-reflex responses. J Appl Physiol 92 : 2309-2318, 2002.

25) Ueno T. Study on relationship between teeth clenching in intercuspal position and isometric movement of upper limbs. J Stomatol Soc 62 : 212-253, 1995. (in Japanese)

26) Sumita Y, Sasaki Y, Ueo T et al. Effect of teeth clenching on force-velocity relationships in isokinetic knee extension. Jpn J Phys Fittness Sports Med $48:$ 365-374, 1999.

27) Sato R, Yamanaka T, Sumita Y et al. Augmentation of eccentric strength of knee extensors with teeth clenching. Jpn J Phys Fitness Sports Med 50: 339-346, 2001.

28) Churei H, Sasaki $Y$, Ueno $T$ et al. Change of grip force production characteristics by teeth clenching. J Jpn Prosthodont Soc 46:732-737, 2002. (in Japanese)

29) Bawa P. Neural control of motor output: Can training change it? Exerc Sport Sci Rev 30:59-63, 2002.

30) Landau WM, Clare MH. Fusimotor function. 4. Reinforcement of the H-reflex in normal subjects. Arch Neurophysiol 46:130-142, 1981.

31) Crone C, Nielsen J. Methodological implication of the postactivation depression of the soleus H-reflex in man. Exp Brain Res 78 : 28-32, 1989.

32) Magladery JW, McDougal DB Jr. Electrophysiological studies of nerve and reflex activity in normal man. I. Identification of H-reflex of certain reflexes in the electromyogram and the conduction velocity of peripheral nerve fibers. Bull Johns Hopkins Hosp 86:265-290, 1950.

33) Rothwell JC, Day BL, Marsden CD et al. Habituation and conditioning of the human long latency stretch reflex. Exp Brain Res 63: 197-204, 1986.

34) Prochazka A. Sensorimotor gain control : A basic strategy of motor system? Prog Neurobiol 33 : 281-307, 1998.

35) Manns A, Miralles R, Guerrero F. The change in electrical activity of the postural muscles of the mandible upon varying the vertical dimension. J Prosthet Dent 45:438-445, 1981.

36) Kohno S, Bando E, Tanaka B et al. A multiple regression analysis for occlusal contact position and masticatory muscle activities. J Jpn Prosthodont Soc $26: 1271-1286,1982$. (in Japanese)

37) Bakke M, Moller E, Thorsen NM. Occlusal contact and maximal muscle activity in natural mandibular positions. J Dent Res 59 (Special issue B) : 892, 1980

38) Jimenez ID. Dental stability and maximal masticatory muscle activity. J Oral Rehabil 14: 591-598, 1987.

39) Carlsson GE, Ingerval B, Kocak G. Effect of increasing vertical dimension on the masticatory system in subjects with natural teeth. J Prosthet Dent $41: 284$, 1979.

40) Al Quran FAM, Lyons MF. The immediate effect of hard and soft splints on the EMG activity of the masseter and temporalis muscle. J Oral Rehabil 26 : 559-563, 1999.

41) Paphangkorakit J, Osborn JW. Effects on human maximum bite force of biting on a softer or harder object. Arch Oral Biol 43 : 833-839, 1998.

42) Sugawara K, Kasai T. Facilitation of motor evoked potentials and H-reflex of flexor Capri radialis induced by voluntary teeth clenching. Human Movement Science 21:203-212, 2002.

43) Boroojerdi B, Battaglia F, Cohen LG et al. Voluntary teeth clenching facilitates human motor system excitability. Clin Neurophysiol 111 : 988-993, 2000.

44) Furubayashi T, Sugawara K, Kasai T et al. Remote effects of self-paced teeth clenching on the excitability of hand motor area. Exp Brain Res 148: 261-265, 2003.

45) Larson CR, Smith A, Luschei ES. Discharge characteristics and stretch sensitivity of jaw muscle afferents in the monkey during controlled isometric bites. J Neurophysiol 46:130-142, 1981.

46) Appenteng K, Lund JP, Seguin JJ. Intraoral mechanoreceptor activity during jaw movement in the anesthetized rabbit. J Neurophysiol 48:27-37, 1982.

47) Trulsson M, Johansson RS. Encoding of amplitude and rate of forces applied to the teeth by human periodontal mechanoreceptive afferents. J Neurophysiol 72 : 1734-1744, 1994. 